\title{
Revisiting extreme precipitation amounts over southern South America and implications for the Patagonian Icefields
}

\section{Tobias Sauter ${ }^{1}$}

$5{ }^{1}$ Climate System Research Group, Institute of Geography, Friedrich-Alexander-University Erlangen-Nürnberg (FAU), Germany

Correspondence to: Tobias Sauter (tobias.sauter@fau.de)

10 Contents of this file

Figures S1 to S3

Tables S1 to S3 


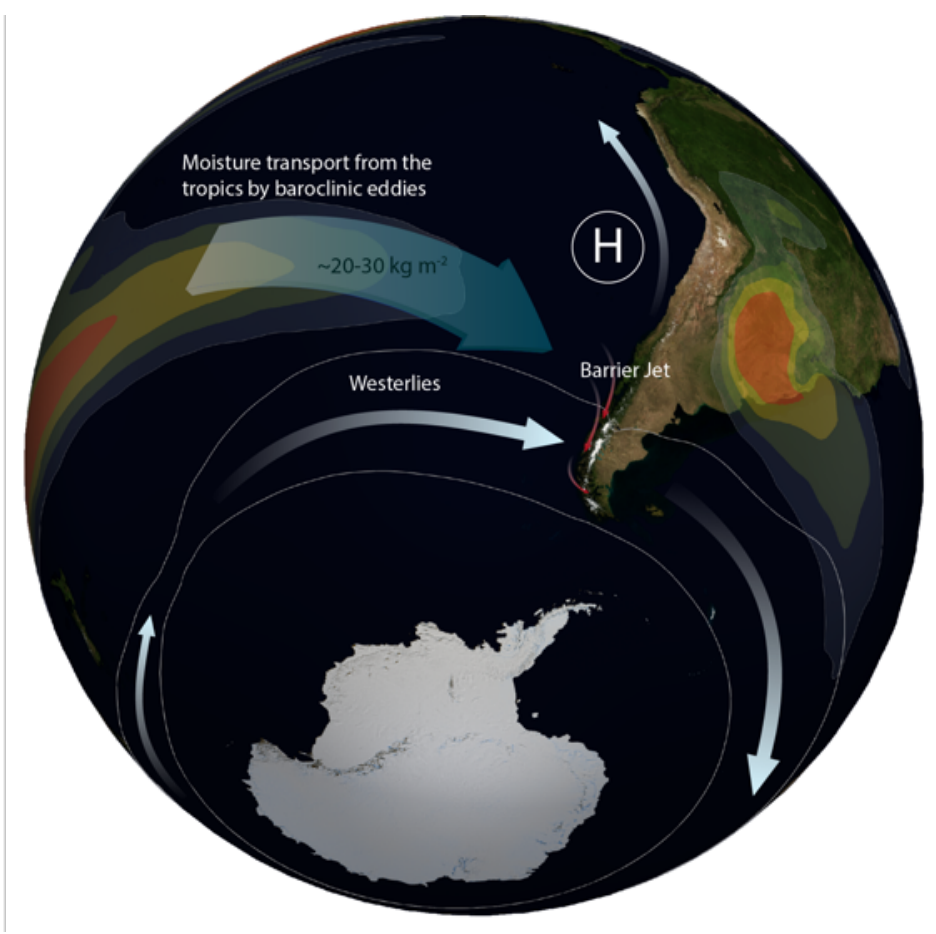

Figure S1: Schematic illustration of the atmospheric large-scale circulation and moisture transport in the South Pacific. Shown are the location of the westerlies for austral summer (December-January-February), the barrier jet along the Andes (red arrows), and the mean moisture transport by baroclinic eddies (blue shaded arrow). The shading indicates regions of high water vapor variability. 


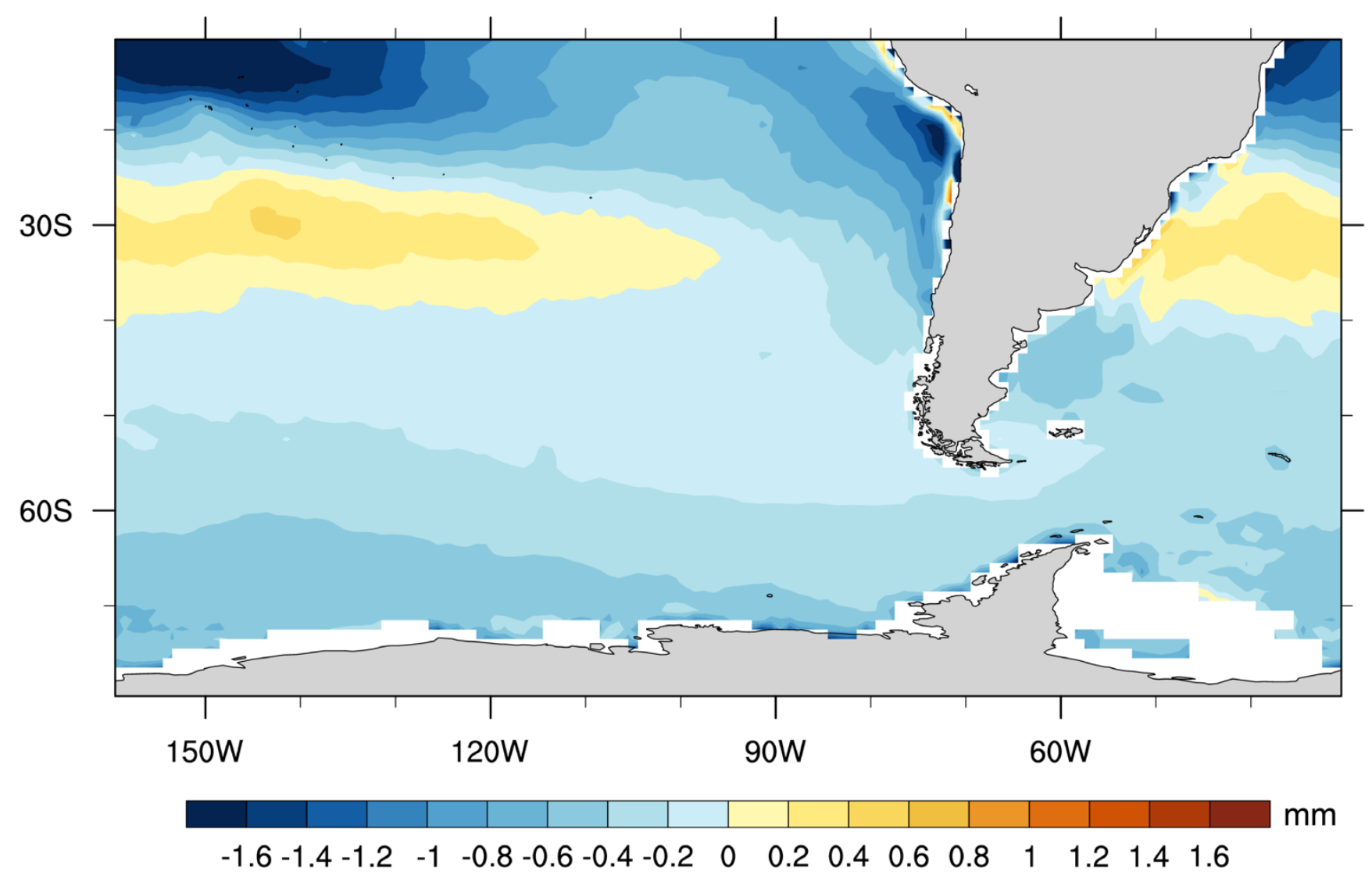

Figure S2: Mean differences in the IWV between ERA Interim and SSMI data for the period 1988-2016. Red shading indicates a positive bias in the ERA Interim data. 


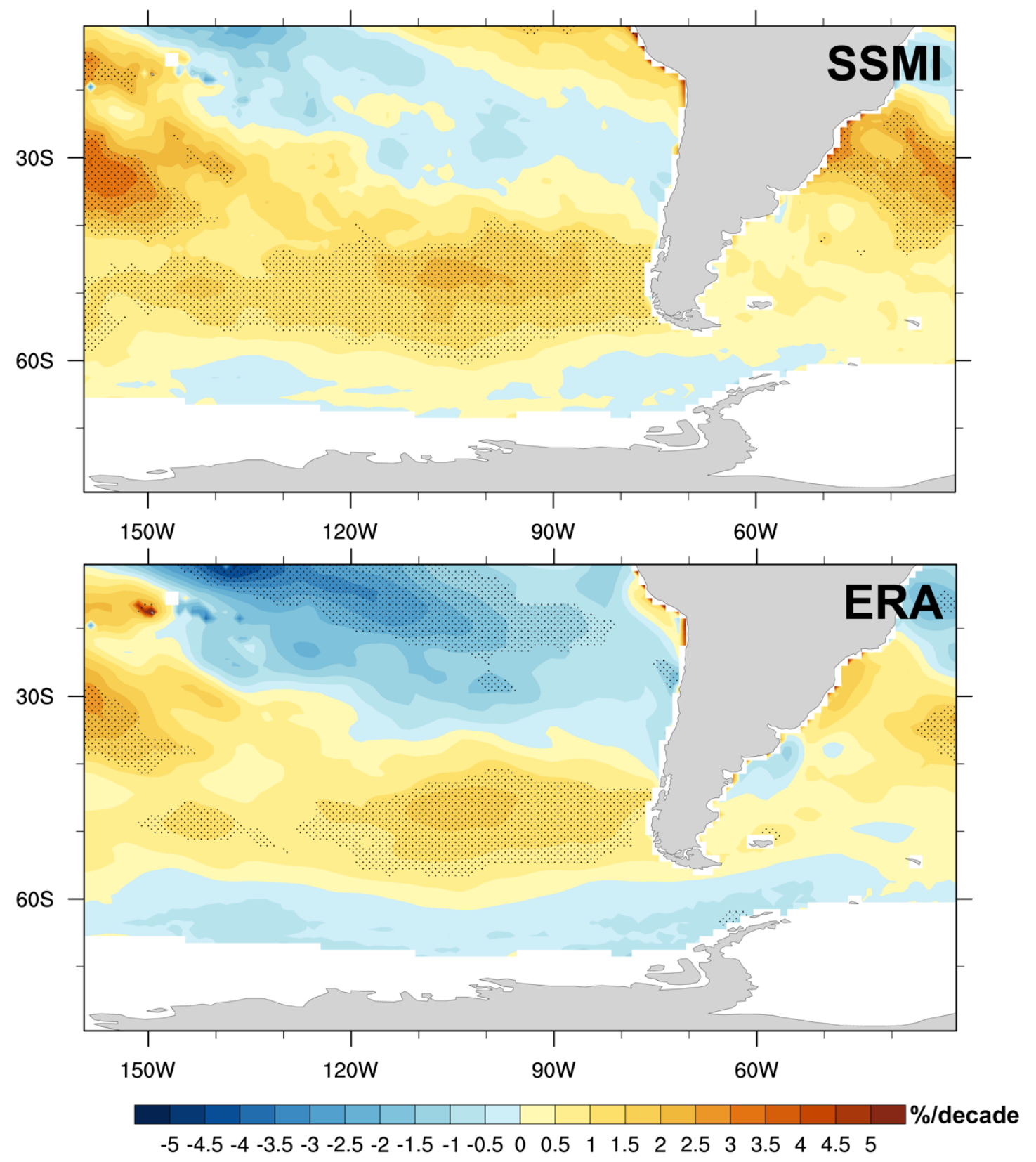

Figure S3: Linear trend of IWV in the SSM/I and ERA-Interim data for the period 1988-2016 (in \% per decade). Dotted areas indicate significant long-term trends $(\mathbf{p}<\mathbf{0 . 0 5})$. 
Table S1. Comparison of the IWV trends between atmospheric soundings and the nearest ERA-Interim grid point. Numbers are given in $\mathrm{mm}_{\text {decade }}{ }^{-1}$. Bold numbers indicate significant trends $(\mathbf{p}<0.05)$.

\begin{tabular}{llll}
\hline & $\mathbf{1 9 8 8 - 2 0 1 6}$ & $\mathbf{1 9 8 8 - 2 0 0 9}$ & $\mathbf{2 0 1 0 - 2 0 1 6}$ \\
\hline Puerto Montt & & & \\
Radiosounding & $-0.22(-1.5 \%)$ & $-0.42(-3.0 \%)$ & $2.87(19.4 \%)$ \\
ERA-Interim & $-0.16(-1.3 \%)$ & $-0.25(-2.0 \%)$ & $0.65(5.2 \%)$ \\
& & & \\
Punta Arenas & & & $1.23(11.3 \%)$ \\
Radiosounding & $0.23(2.2 \%)$ & $0.07(0.6 \%)$ & $0.80(7.0 \%)$ \\
ERA-Interim & $0.14(1.3 \%)$ & $0.05(0.4 \%)$ & \\
\hline
\end{tabular}

5 Table S2. Comparison of the OPM ( $D R=0.45)$ with observations. Given are the latitude (lat), longitude (lon), altitude (alt), the precipitation values from the orographic precipitation model (OPM) and the observations (Obs) at the weather stations The precipitation values are given in $\mathrm{mm} \mathrm{yr}^{-1}$.

\begin{tabular}{lccccr}
\hline Location & lat & lon & alt & OPM & Obs \\
\hline Villa Cerro Castillo & -46.12 & -72.15 & 345 & 471.30 & 282.45 \\
Rio Ibaez En Desembocadura & -46.26 & -71.99 & 220 & 298.12 & 623.58 \\
Bahia Murta & -46.46 & -72.66 & 240 & 849.30 & 1017.93 \\
Lago General Carrera Fachinal & -46.54 & -72.22 & 18 & 579.54 & 333.60 \\
Glaciar San Rafael & -46.64 & -73.85 & 8 & 3829.02 & 1271.60 \\
Puerto Guadal & -46.84 & -72.70 & 210 & 745.45 & 656.28 \\
Estancia Valle Chacabuco & -47.11 & -72.48 & 343 & 613.92 & 159.60 \\
Rio Nef Antes Junta Estero El Revalse & -47.13 & -73.08 & 281 & 895.86 & 974.88 \\
Rio Baker En Angostura Chacabuco & -47.14 & -72.72 & 160 & 673.35 & 856.73 \\
Lago Cachet 2 En Glaciar Colonia & -47.19 & -73.25 & 427 & 1088.06 & 243.25 \\
Lord Cochrane Ad. & -47.24 & -72.58 & 204 & 744.24 & 652.65 \\
Rio Cochrane En Cochrane & -47.25 & -72.56 & 140 & 788.03 & 514.75 \\
Rio Colonia En Nacimiento & -47.33 & -73.11 & 146 & 986.30 & 1261.80 \\
Caleta Tortel & -47.79 & -73.53 & 10 & 2389.95 & 1870.28 \\
Rio Pascua Ante Junta Rio Quetru & -48.15 & -73.08 & 20 & 1668.09 & 2137.12 \\
Lago Ohiggins En Villa Ohiggins & -48.51 & -72.59 & 300 & 752.40 & 909.18 \\
Candelario Mancilla & -48.87 & -72.73 & 300 & 850.18 & 519.25
\end{tabular}




\begin{tabular}{lrrrrr} 
Rio Punta Eva En Puerto Eden & -49.11 & -74.41 & 10 & 3607.47 & 2840.15 \\
El Calafate Aero & -50.26 & -72.05 & 204 & 433.19 & 149.60 \\
Lago Dickson & -50.82 & -73.11 & 200 & 1301.05 & 1130.96 \\
Lago Paine & -50.84 & -72.90 & 440 & 1132.83 & 500.30 \\
Cerro Guido & -50.89 & -72.33 & 230 & 820.83 & 312.50 \\
Amalia & -50.95 & -73.69 & 0 & 4350.81 & 2801.60 \\
Rio Paine En Parque Nacional 2 & -50.96 & -72.79 & 90 & 1139.24 & 724.67 \\
Nunatak Grey & -50.97 & -73.22 & 300 & 1414.56 & 589.70 \\
Lago Sarmiento & -51.01 & -72.71 & 110 & 1111.92 & 352.85 \\
Lago Pehoe & -51.07 & -72.99 & 40 & 1347.67 & 868.18 \\
Lago Grey & -51.11 & -73.13 & 50 & 1397.74 & 663.07 \\
Glaciar Tindall & -51.11 & -73.28 & 345 & 1584.05 & 1200.28 \\
Torres Del Paine & -51.18 & -72.96 & 25 & 1431.40 & 750.34 \\
Rio Rincon En Ruta Y-290 & -51.31 & -72.82 & 36 & 1554.36 & 769.70 \\
Rio Serrano En Desembocadura & -51.33 & -73.10 & 25 & 1671.86 & 1227.01 \\
\hline
\end{tabular}

Table S3. Summary of the WRF configuration.

Value

\section{Domain configuration}

Horizontal grid spacing

$12.5-\mathrm{km}, 2.5-\mathrm{km}$, and $500-\mathrm{m}$

Vertical levels

55

Model top pressure

$100 \mathrm{hPa}$

\section{Model physics}

Radiation

RRTMG

Microphysics

Morrison

Cumulus

Kain-Fritsch

Planetary boundary layer

MYNN Level 2.5

Atmospheric surface layer

Monin Obukhov

Land surface

Noah-MP

Top boundary condition

Rayleigh damping 
Forcing

ERA-Interim

$0.75^{\circ} \times 0.75^{\circ}, 6$-hourly 